\title{
A Study of Contrast Enhancement by Image Fusion using Edge Detection Techniques
}

\author{
Pallabi Ghosh \\ $4^{\text {th }}$ year, B.Tech \\ Institute of Engineering \& \\ Management
}

\author{
Debanjana Dasgupta \\ $4^{\text {th }}$ year, B.Tech \\ Institute of Engineering \& \\ Management
}

\author{
Debalina Ghosh \\ Asst. Professor \\ Institute of Engineering \& \\ Management
}

\begin{abstract}
There are various methods available in the literature for improving the visual quality of an image. Contrast enhancement of an image by histogram equalization is one such technique. But histogram equalization alone results in data loss and also the mean brightness of the resultant output image approaches to the middle gray level. In this paper, the edge strengths of the original and the equalized images have been found by four edge detection algorithms, Robert, Sobel, Prewitt and Canny separately and then depending on their edge strengths and following a simple equation, fusion of the two images are done. These resulting fused images contain all the useful data of the original image as well as their contrasts are enhanced. The Standard Deviation and Gradient of the respective fused images are then compared and it is found that for almost all images image fusion using Canny edge detector as a filter yields best result.
\end{abstract}

\section{General Terms}

Image Processing, Contrast Enhancement, Histogram Equalization, Edge Detection, Image Fusion

\section{Keywords}

Contrast Enhancement, Histogram equalization, Standard Deviation, Average Gradient, Robert, Sobel, Prewitt, Canny, Image Fusion.

\section{INTRODUCTION}

In computer vision and image processing, the main goal of image enhancement is to process the input image in such a way that the output image is more suitable [1] for interpretation by the humans as well as by machines. Histogram equalization is a technique for image enhancement but most images taken from scenes with non-uniform distributed illumination show the problem of being too contrasty [2][3]. Image fusion, on the other hand, is the process of combining relevant information from two or more images into a single image. The resulting image will be more informative than any of the input images and also its contrast and clarity will be enhanced. With the advancement of technology image fusion has important application in the field of medical imaging, microscopic imaging, remote sensing, computer vision, and robotics. In this paper, linear image fusion [4] of the original and the equalized image is done so that the output fused image has all the useful information of the original image and its contrast is also enhanced because of its fusion with the equalized image. During fusion different edge detection operators like Roberts, Sobel, Prewitt and Canny are used separately as a filter [2] and the visual qualities of the output fused images are studied and compared using statistical parameters Standard Deviation and Average Gradient.

\section{HISTOGRAM EQUALIZATION}

A Histogram of an image offers a graphical representation of the tonal distribution of the gray values in a digital image. Histogram equalization spreads out the intensity values along the total range of gray level values. It is, therefore, a technique for adjusting image intensities to enhance contrast [1][3]. The method is useful in images with backgrounds and foregrounds that are both bright or both dark [1]. A disadvantage of this method is while increasing the contrast of its background, the signal gets distorted, it may increase the contrast of background noise and some images may become too much contrasty. [3][5]

\section{METHODOLOGY}

\subsection{Image Quality Measures}

\section{A. Standard Deviation}

Standard deviation is the deviation about mean. It represents the dynamic range of values present in an image about the mean [16]. Greater is the standard deviation greater is the contrast of the fused image. If $m$ is the mean of the image, then the standard deviation about the mean is given by:

$$
S T D=\sqrt{\frac{\sum_{x=0}^{M-1} \sum_{y=0}^{N-1}(I(x, y)-m)^{2}}{M N}}
$$

Here, $\mathrm{I}(\mathrm{x}, \mathrm{y})$ is the intensity of the pixel, $\mathrm{M}$ is the number of rows and $\mathrm{N}$ is the number of columns [16].

B. Average Gradient

Average gradient is used for measuring the clarity of the image.[16] More the average gradient more is the clarity of the image. Average Gradient is given by the formula:

$$
A G=\frac{\sum_{x=0}^{M-1} \sum_{y=0}^{N-1} g(x, y)}{M N}
$$

Here, $\mathrm{g}(\mathrm{x}, \mathrm{y})$ is the magnitude gradient at location ( $\mathrm{x}$, y)[16].

\subsection{Image Fusion}

Image Fusion is a process of combining a set of images into a single image which is more informative and complete than any of the input images. Image fusion techniques can improve the contrast and clarity of the image. Similarly lower resolution multispectral images can be fused with higher resolution panchromatic images to get high resolution images which can provide insightful information about the scene under consideration. 
A general expression to obtain a linearly fused image $I_{f}$ with two source images is given as:

$$
\mathrm{I}_{\mathrm{f}}=\alpha * \mathrm{I}+(1-\alpha) * \mathrm{I}_{\mathrm{h}}
$$

where $I$ and $I_{h}$ are the original and histogram equalized image respectively and $0 \leq \alpha \leq 1$ is a scaling factor defined by the user.[4]

\subsection{Edge Detection}

Edge detection refers to the process of identifying and locating sharp discontinuities in an image. The discontinuities are abrupt changes in pixel intensity which characterize boundaries of objects in a scene. Classical methods of edge detection involve convolving the image with an operator, which is constructed to be sensitive to large gradients in the image while returning values of zero in uniform regions. There are an extremely large number of edge detection operators available, each designed to be sensitive to certain types of edges [6]. In this paper we have used Roberts, Sobel, Prewitt and Canny operators as filters during image fusion and the resulting fused image is studied.

\subsection{Image Fusion using Edge Detection}

Some images are often represented by close contrast values. As a result of which it becomes difficult to distinguish two adjacent objects of nearly similar intensities in the original image. But due to uniform spreading of intensities in the histogram equalized image, sometimes the boundary between the said adjacent objects becomes distinguishable. Again, due to data loss sometimes an edge which is distinguishable in the original image remains unidentified in the equalized image. So, we are applying edge detectors to both the images and calculating the edge strengths. The edge strength of each image is then used to determine the weightage of each pixel of the images using the function:

$$
\alpha=1 /\left(1+10_{1}^{\left(-\left(\mathrm{S}_{1}(\mathrm{x}, \mathrm{y})-\mathrm{S}_{2}(\mathrm{x}, \mathrm{y})\right)\right)}\right)
$$

where $S_{1}(x, y)$ is the edge strength of the original image and $\mathrm{S}_{2}(\mathrm{x}, \mathrm{y})$ is the edge strength of the equalized image.[2]

This function actually represents three cases which are as follows:

Case-1: When an edge is present in the original image and not in the equalized image i.e. $S_{1}(x, y)>S_{2}(x, y)$, then we take the pixel value of the original image[2][4].

Case-2: When an edge is present in the equalized image and not in the original image i.e. $S_{2}(x, y)>S_{1}(x, y)$, we take the pixel value of the equalized image[2][4].

Case-3: When the edge is either present or absent in both the original and equalized image i.e. $S_{1}(x, y)=S_{2}(x, y)$, then we take the average of the pixel values of both the images[2][4].

Now, the value of $\alpha$ is used in the following equation to get the fused image:

$$
\mathrm{I}_{\mathrm{f}}=\mathrm{I}_{1} * \alpha+(1-\alpha) * \mathrm{I}_{\mathrm{h}}
$$

where $I_{f}$ is the fused image, $I_{1}$ is the original image and $I_{h}$ is the histogram equalized image[2][4].
The better the edge detection operator, the better will be the visual quality of the resultant fused image.

\subsubsection{Fusion Using Roberts Operator}

The Roberts Cross operator performs a simple, quick to compute, 2-D spatial gradient measurement on an image[8][9] Pixel values at each point in the output is the estimated absolute magnitude of the spatial gradient of the input image at that point[8][10]. It thus highlights the region of high spatial frequency which mainly corresponds to edges [8][11]. The convolution kernels used shown in Figure 1. One kernel is simply the other rotated by $90^{\circ}$ [6][8]. The main advantages of this operator is that it is very quick to compute and computation contains mainly addition and subtraction.[8] Moreover, there are no parameters to set. But the disadvantages are it is sensitive to noise and produces very weak responses to genuine edges [8].

\begin{tabular}{|c|c|}
\hline+1 & 0 \\
\hline 0 & -1 \\
\hline \multicolumn{2}{|c|}{$\mathrm{G}_{\mathrm{x}}$}
\end{tabular}

\begin{tabular}{|c|c|}
\hline 0 & +1 \\
\hline-1 & 0 \\
\hline \multicolumn{3}{|c|}{$\mathrm{G}_{\mathrm{y}}$} \\
\hline
\end{tabular}

Fig 1: masks used for Roberts operator

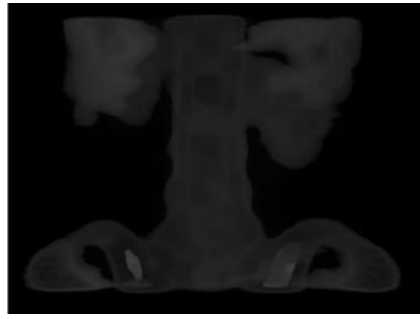

(a)

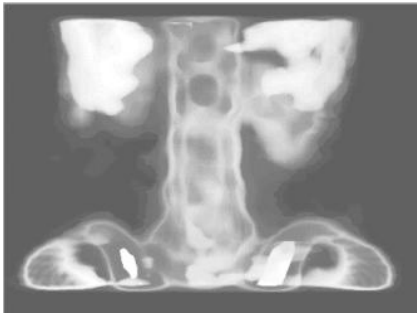

(b)

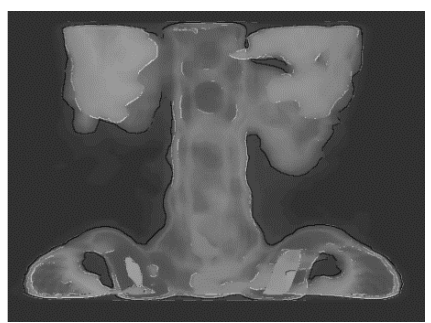

(c)

Fig 2: Spine- (a) original image, (b) equalized image, and (c) fused image using Roberts operator

\subsubsection{Fusion Using Sobel Operator}

This operator is similar to Roberts operator but with $3 \times 3$ convolution kernels as shown below [12]. Because of such kernels edges running horizontally and vertically to the pixel grid are maximally recognized[12][13].The Sobel operator is slower than the Roberts Operator but it is less sensitive to noise as its larger convolution kernel smoothes the image.[10] But again, due to this smoothing natural edges in images often leads to lines in the output image.[13][6] 


\begin{tabular}{|l|l|l|}
\hline-1 & 0 & +1 \\
\hline-2 & 0 & +2 \\
\hline-1 & 0 & +1 \\
\hline \multicolumn{4}{|c|}{$\mathrm{G}_{\mathrm{x}}$}
\end{tabular}

\begin{tabular}{|c|c|c|}
\hline+1 & +2 & +1 \\
\hline 0 & 0 & 0 \\
\hline-1 & -2 & -1 \\
\hline \multicolumn{4}{|c}{$\mathrm{G}_{\mathrm{y}}$} \\
\end{tabular}

Fig 3: Mask used by Sobel Operator

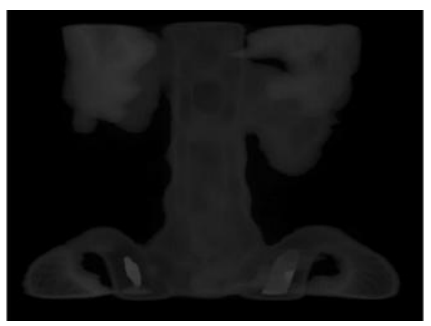

(a)

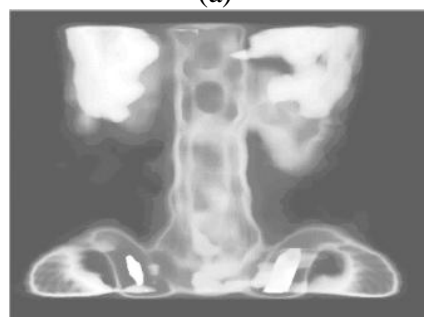

(b)

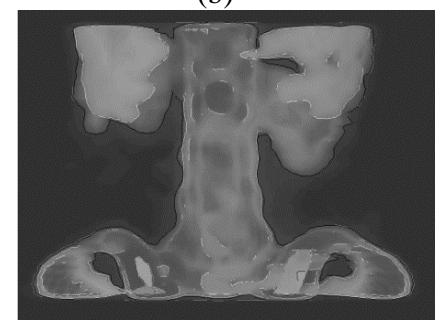

(c)

Fig 4: Spine- (a) original image, (b) equalized image, and (c) fused image using Sobel operator

\subsubsection{Fusion Using Prewitt Operator}

Prewitt operator is similar to the Sobel operator and is used for detecting vertical and horizontal edges in images[6]. But, unlike Sobel operator, this operator does not put any emphasis on pixels that are closer to the center of the masks[7].The operation mainly outputs two images, one estimating the local edge gradient magnitude and one estimating the edge orientation of the input image[15]. In our paper we have used the kernel shown in fig 5[6][7].

\begin{tabular}{|c|c|c|}
\hline-1 & 0 & +1 \\
\hline-1 & 0 & +1 \\
\hline-1 & 0 & +1 \\
\hline \multicolumn{4}{|c|}{$\mathrm{G}_{\mathrm{x}}$} \\
\hline
\end{tabular}

\begin{tabular}{|c|c|c|}
\hline+1 & +1 & +1 \\
\hline 0 & 0 & 0 \\
\hline-1 & -1 & -1 \\
\hline \multicolumn{4}{|c|}{$\mathrm{G}_{\mathrm{y}}$}
\end{tabular}

Fig 5: Mask used by Prewitt Operator

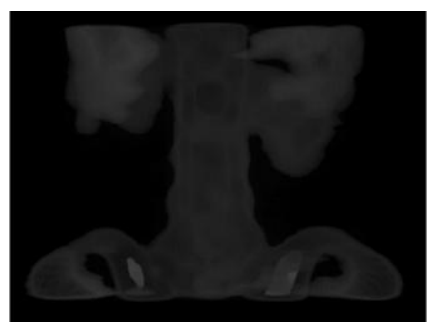

(a)

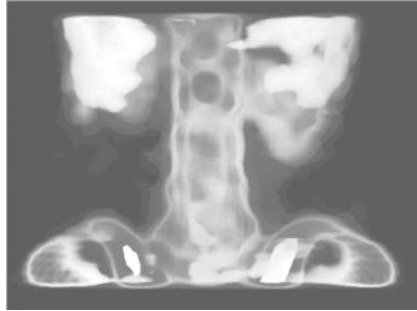

(b)

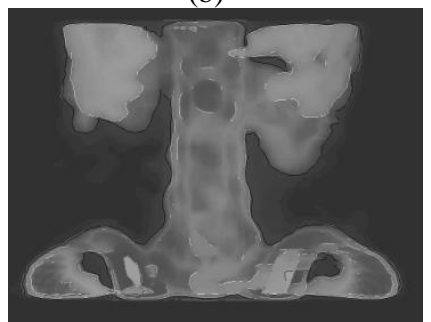

(c)

Fig 6: Spine- (a) original image, (b) equalized image, and (c) fused image using Prewitt operator

\subsubsection{Fusion Using Canny Operator}

Canny operator is an optimal edge detector which works in a multistage process. The stages include smoothing phase, highlighting regions with high spatial derivatives phase, application of non-maximum suppression phase, threshold application phase and tracking edge by hysteresis phase.[6][10]. This is an operator where width of the Gaussian kernel used in the second phase can be increased to reduce the detector's sensitivity to noise, at the expense of losing some of the finer details in the image.[10][14] But one problem with this operator is that place where three ridges meet, the tracker will treat two of the ridges as single line statement and the third one as the line approaching that does not quite connect[14].

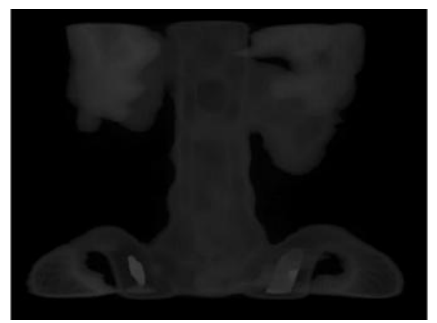

(a)

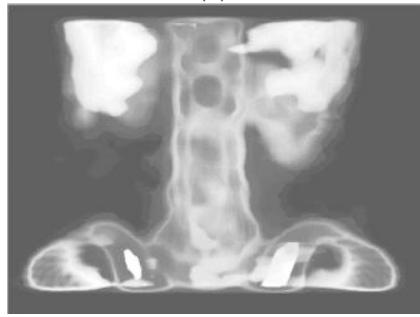

(b)

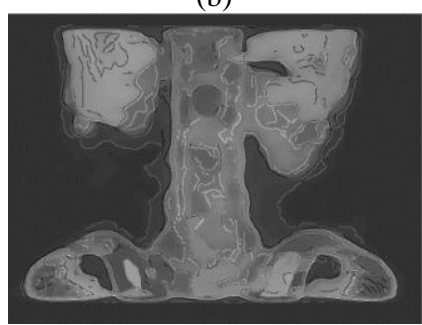

(c)

Fig 7: Spine- (a) original image, (b) equalized image, and (c) fused image using Canny operator 


\section{RESULTS}

The aforementioned ways of image fusion using different edge detection techniques have been applied to different images in this section. Here, we use standard deviation and average gradient of an image as parameters to compare the different results which have been yielded after applying image fusion on them. The results are shown in Fig. 8,9,10 and Tables 1, 2. All the simulation has been carried out in MATLAB.

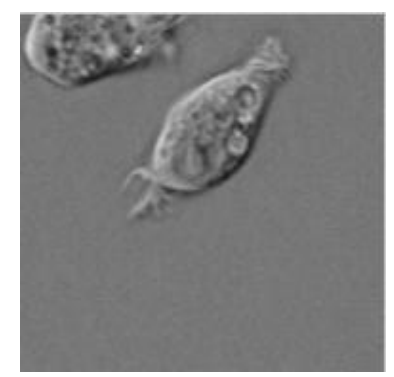

(a)

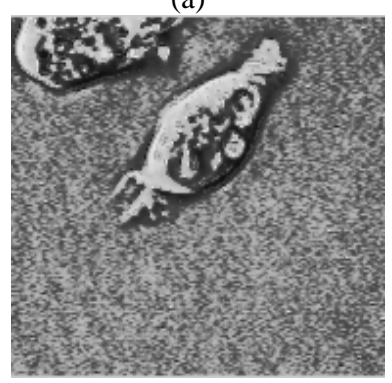

(c)

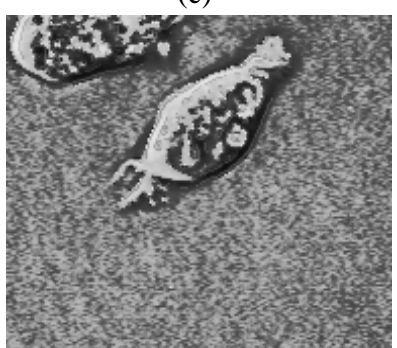

(e)

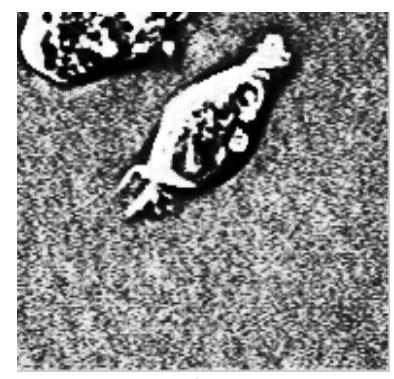

(b)

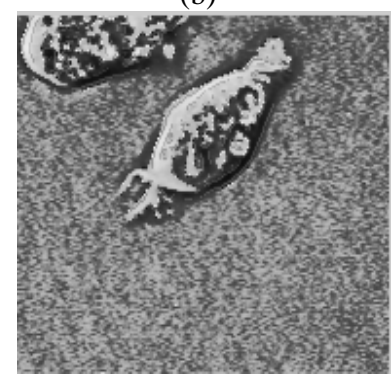

(d)

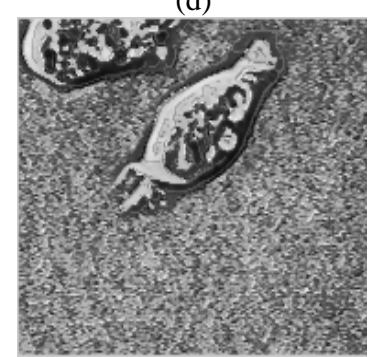

(f)
Fig 8: cell- (a) original Image, (b) equalized image, (c) fused image using Roberts, (d) fused image using Prewitt, (e) fused image using Sobel operators, and (f) fused image using Canny operators

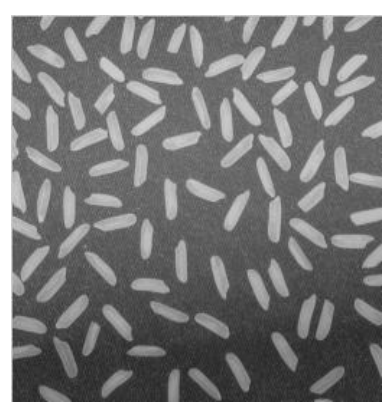

(a)

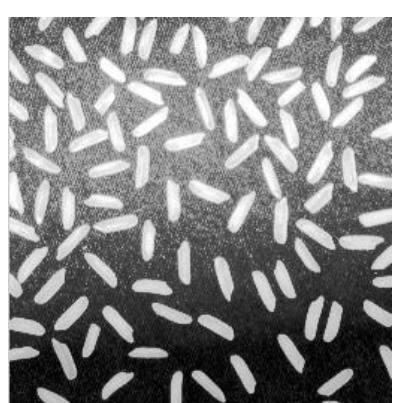

(b)

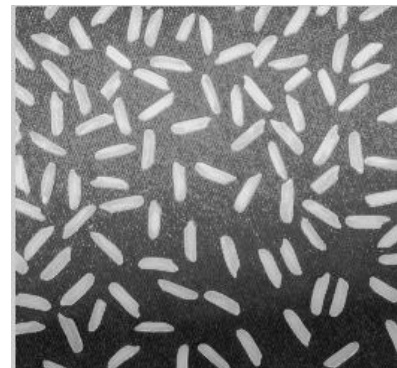

(b)

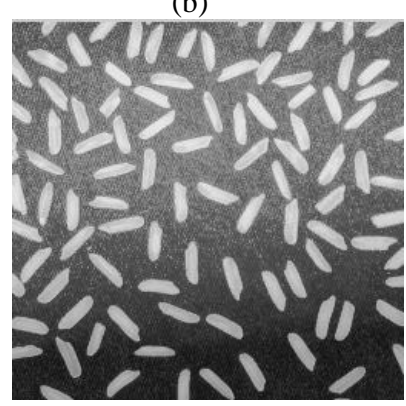

(e)

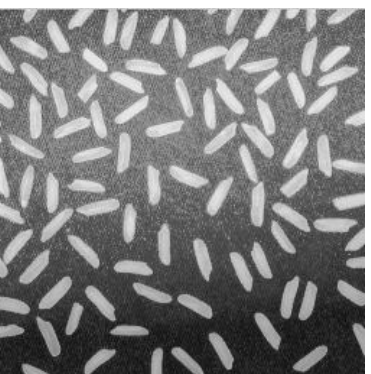

(d)

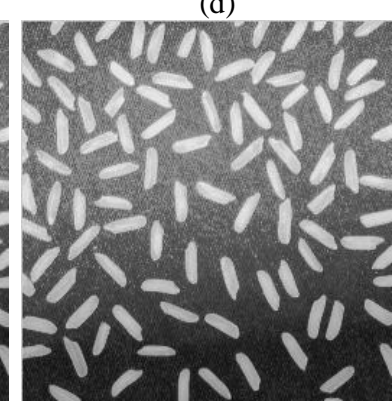

(f)

Fig 9: Rice - (a) Original image, (b) Equalized image, (c) Fused image using Roberts, (d) Fused image using Prewitt,

(e) Fused image using Sobel Operators, and (f) fused image using Canny operators

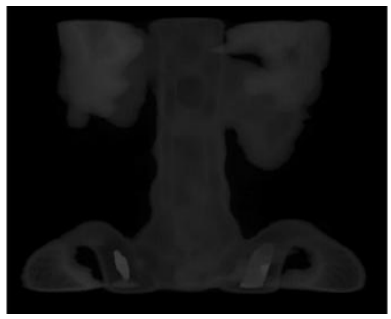

(a)

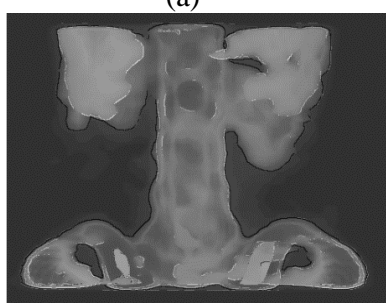

(c)

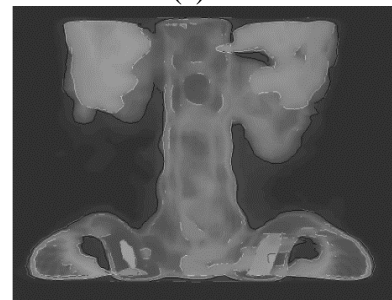

(e)

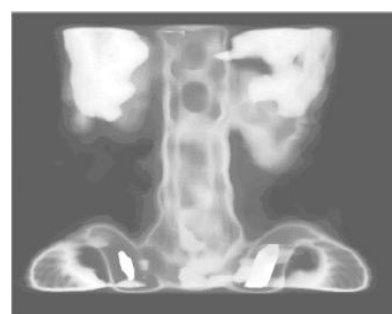

(b)

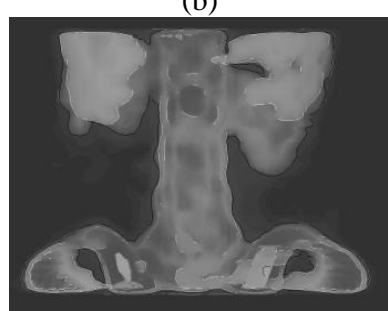

(d)

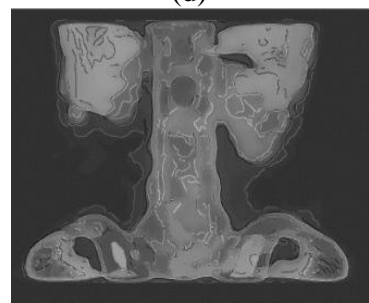

(f)

Fig 10: Spine - (a) Original image, (b) Equalized image, (c) Fused image using Roberts, (d) Fused image using Prewitt, (e) Fused image using Sobel Operators, and (f) fused image using Canny operators 
Table 1: Comparison between the standard deviations of the different images when fused using different edge detectors

\begin{tabular}{|c|l|l|l|}
\hline & CELL & RICE & SPINE \\
\hline $\begin{array}{c}\text { ORIGINAL } \\
\text { IMAGE }\end{array}$ & 13.8616 & 42.4935 & 16.6426 \\
\hline $\begin{array}{c}\text { EQUALIZED } \\
\text { IMAGE }\end{array}$ & 74.7378 & 74.8673 & 53.2791 \\
\hline $\begin{array}{c}\text { FUSED IMAGE } \\
\text { USING } \\
\text { ROBERTS }\end{array}$ & 41.7430 & 57.7212 & 36.8619 \\
\hline $\begin{array}{c}\text { FUSED IMAGE } \\
\text { USING SOBEL }\end{array}$ & 42.2083 & 57.7388 & 36.7500 \\
\hline $\begin{array}{c}\text { FUSED IMAGE } \\
\text { USING } \\
\text { PREWITT }\end{array}$ & 42.1976 & 57.7354 & 36.7121 \\
\hline $\begin{array}{c}\text { FUSED IMAGE } \\
\text { USING CANNY }\end{array}$ & 46.4468 & 58.1651 & 37.9226 \\
\hline
\end{tabular}

Table 2: Comparison between the average gradients of the different images when fused using different edge detectors

\begin{tabular}{|c|l|l|l|}
\hline & CELL & RICE & SPINE \\
\hline $\begin{array}{c}\text { ORIGINAL } \\
\text { IMAGE }\end{array}$ & 3.0781 & 7.6617 & 0.5207 \\
\hline $\begin{array}{c}\text { EQUALIZED } \\
\text { IMAGE }\end{array}$ & 34.8598 & 16.2701 & 2.0216 \\
\hline $\begin{array}{c}\text { FUSED IMAGE } \\
\text { USING } \\
\text { ROBERTS }\end{array}$ & 19.1982 & 11.5366 & 2.4593 \\
\hline $\begin{array}{c}\text { FUSED IMAGE } \\
\text { USING SOBEL }\end{array}$ & 19.5476 & 11.4666 & 2.4682 \\
\hline $\begin{array}{c}\text { FUSED IMAGE } \\
\text { USING } \\
\text { PREWITT }\end{array}$ & 19.5317 & 11.4695 & 2.4411 \\
\hline $\begin{array}{c}\text { FUSED IMAGE } \\
\text { USING CANNY }\end{array}$ & 22.0585 & 11.4562 & 4.4258 \\
\hline
\end{tabular}

\section{CONCLUSION}

Comparing the simulation results, we observe that the operator, using which the image fusion will yield best result, is mainly dependent on the characteristics of the image. But it is also observed that image fusion using Canny operator gives best results in most of the cases. Depending on purpose and constraints one of the techniques can be used in various medical imaging projects. For example prostate of a human contains different layers having almost equal density. So the image that we get after prostate segmentation is not clear. To distinguish each of these layers we may fuse the image using canny operator and thus increase the clarity of the image to the maximum.

\section{REFERENCES}

[1] R. Gonzalez and R. Woods, Digital Image Processing, 2nd ed., Prentice Hall, Jan. 2002).

[2] Xiaoying Fang, Jingao Liu, Wenquan Gu, Yiwen Tang, "A Method to Improve the Image Enhancement Result based on Image Fusion", International Conference on Multimedia Technology, 2011, pp. 55-58

[3] S.-D. Chen, A. Ramli, "Contrast enhancement using recursive mean-separate histogram equalization for scalable brightness preservation," IEEE Trans. On Consumer Electronics, vol. 49, no. 4, pp. 1301-1309, Nov.2003.

[4] Cheng-Hsiung Hsieh, Bo-Chang Chen, Chih-Ming Lin, Qiangfu Zhao, "Detail Aware Contrast Enhancement with Linear Image Fusion", International Symposium on Aware Computing, 2010,pp.1-5

[5] Y.-T. Kim, "Contrast enhancement using brightness preserving bi - histogram equalization", IEEE Trans. On Consumer electronics, vol. 43, no. 1, pp. 1-8, Feb. 1997.

[6] Raman Maini,Dr. Himanshu Aggarwal, "Study and Comparison of Various Image Edge Detection Techniques", International Journal of Image Processing (IJIP), Volume (3) : Issue (1)

[7] [7] Seif, A.,et.al. ;“A hardware architecture of Prewitt edge detection", Sustainable Utilization and Development in Engineering and Technology (STUDENT), 2010 IEEE Conference, Malaysia, pp. 99 101, 20-21 Nov. 2010.

[8] http://homepages.inf.ed.ac.uk/rbf/HIPR2/roberts.htm

[9] Y.Ramadevi, T.Sridevi, B.Poornima, B.Kalyani, "SEGMENTATION AND OBJECT RECOGNITION USING EDGE DETECTION TECHNIQUES", International Journal of Computer Science \& Information Technology, Vol. 2, No 6, December 2010.

[10] Bhavya R, Vijay V, "Edge Detection and Object Extraction Using Unbalanced Weights for Foreground and Background", International Journal of Scientific and Research Publications, Volume 2, Issue 12, December 2012.

[11] www.robotics.technion.ac.il/courses/advanced.../arl_7

[12] J. Matthews. "An introduction to edge detection: The sobel edge detector," Available at http://www.generation5.org/content/2002/im01.asp, 2002.

[13] http://homepages.inf.ed.ac.uk/rbf/HIPR2/sobel.htm

[14] http://homepages.inf.ed.ac.uk/rbf/HIPR2/canny.htm

[15] http://homepages.inf.ed.ac.uk/rbf/HIPR2/prewitt.htm

[16] V.Saini, T.Gulati, "A Comparative Study on Image Enhancement Using Image Fusion", IJARCSSE, Vol. 2,No.10,October2012 